\title{
Assessing and Managing Delirium in Surgical and Internal Medicine: an Overview
}

İsmet KIRPINAR

Department of Psychiatry, Bezmialem Vakıf University School of Medicine, İstanbul, Turkey

\begin{abstract}
Delirium is most common neuropsychiatric disorder that affects patients in surgical and internal medicine clinics. It is defined as a transient, usually reversible organic mental disorder that has a rapid onset and fluctuating course. Clinically, delirium is an acute or subacute organic mental syndrome that is characterized by a disturbance in attention (reduced ability to direct, focus, sustain, and shift attention), awareness (reduced orientation to the environment), and other cognitive abilities (e.g., memory deficit and disorientation) and is not better explained by preexisting, established, or other evolving neurocognitive disorders. Studies have found that between $10 \%$ and $20 \%$ of all adult inpatients are diagnosed with delirium and that it occurs in up to $50 \%$ of elderly inpatients. Delirium itself is not a disease but rather a clinical syndrome, which results from an underlying disease. There is evidence from the history, physical examination, or laboratory findings that the disturbance is a physiological consequence of an underlying medical condition, substance intoxication or withdrawal, use of a medication, or a toxin exposure, or it is a combination of these factors. Accurate identification of underlying causes and risk factors is essential in early stage diagnosis and treatment. Treatment of delirium requires the treatment of the underlying causes. Psychotropic medications are used to comfort patients or enable better patient management. This study aims to review the clinical characteristics, etiological and risk factors, and different methods to obtain an accurate diagnosis and treatment of delirium.
\end{abstract}

Keywords: Delirium, risk factors, delirium management, treatment

\section{Introduction}

Delirium is a syndrome that causes acute disorders in brain functions, which can be developed in a short time period because of its numerous etiologies. It manifests itself through various cognitive disorders, mainly in consciousness. Diagnostic symptoms are attention and awareness deficits, accompanied by other cognitive disorders such as memory and orientation deficits (1). It is the most common acute disorder that affects hospitalized adult patients. It is reported to develop in $10 \%-20 \%$ of all hospitalized adult patients, in $30 \%-40 \%$ of hospitalized elderly patients, and in up to $80 \%$ of intensive care patients $(2,3)$. These high prevalence rates, along with its distinctive negative effects on factors such as medical morbidity, hospitalization duration, and post-discharge progression increase the importance of diagnosing and treating delirium $(4,5)$. Delirium represents a red flag that informs of the severity of the medical condition that causes it and suggests that the condition is at a stage in which the mortality/morbidity rates could increase.

Delirium is defined as a temporary and reversible brain disorder. It is not a disease; it is a syndrome that is caused by certain clustering symptoms and develops beause of various causes. Diagnosis is overlooked in many cases, or it can erroneously be assessed as dementia, depression, mania, or a normal consequence of old age.

It has been known since ancient times that poisoning, fever, and other medical/neurological conditions cause sudden behavioral and consciousness changes. Presentation of such cases existed in Celsus, who coined the term delirium from "de lira" (to go off the path) in the first century; the term was found in the writings of Galen and Hippocrates. The syndrome, which until the $18^{\text {th }}$ century was referred to as phrenitis, frenziness, and febrile insanity, started to be used for cognitive disorders in the $19^{\text {th }}$ century, and Sutton defined "delirium tremens" in 1813. In 1881, Wernicke described the encephalopathy named after him. The current concept of delirium was formed on the basis of the works of Lipowski in the 
1960 s and with the contribution of others, and the diagnostic criteria were clarified. There is still confusion regarding the name of this syndrome, which has been referred to with various synonymous terms (acute organic psychosis, metabolic encephalopathy, acute confusional state, toxic encephalopathy, and acute organic brain syndrome). Some of these terms are still used, especially in non-psychiatric disciplines (3-6).

Delirium has various definitions in diagnostic systems, notably in the Diagnostic and Statistical Manual of Mental Disorders (DSM) (1). However, all of these definitions include the following core symptoms: 1) awareness deficits accompanied by attention deficits; 2) cognitive changes (orientation and memory deficits), and 3) tendency to develop within dayshours and to progress.

\section{Clinical and Research Effects}

Prevalence: The point prevalence of delirium in the general population is reported to be $0.4 \%$ for those aged $\geq 18$ years and $1.1 \%$ for those aged $\geq 55$ years. It is stated that $60 \%$ of all elderly people in nursing homes suffer from delirium at one point. It can also occur in $10 \%-20 \%$ of all adults who are hospitalized because of medical conditions and in up to $80 \%$ of all intensive care patients (2). It has been emphasized that these rates are much higher for some clinical conditions. Advanced age and the presence of comorbidities are among the most prominent risk factors. In a study comprising 47 patients who underwent vascular surgery, it was reported that $36.2 \%$ of the patients developed delirium, with the need for transfusion being increased and metabolic stability being harder to restore in these patients (7). In two studies that investigated the risk factors for the development of delirium in cardiac patients, the prevalence of delirium in the highrisk group, namely the advanced age group, was found to be $11.4 \%$ and $13.5 \%(8,9)$. In a prospective study involving patients with late-stage cancer, which can be accompanied by numerous medical conditions, 32\% of patients developed delirium (10). In a study involving 717 nursing home patients aged $\geq 75$ years, delirium was observed in $43.9 \%$ of patients, and coexistence of delirium and dementia was observed in $18.8 \%$ of patients (11). In another study involving 225 patients from geriatric care centers, it was reported that $18 \%$ of patients were diagnosed with delirium during admittance and $24 \%$ developed delirium during admittance (12). Delirium is reported to be more common among Caucasians and in women than in men. It can occur at any age, but it mostly develops in old people and people with impaired cognitive function. Thus, dementia constitutes the highest risk condition. Delirium because of general medical conditions is more common in children and individuals aged $>60$ years. Delirium cases because of alcohol and substance intoxication or withdrawal effects are more commonly observed from adolescence to late thirties.

Mortality/Morbidity: Development of delirium increases the mortality rates of severely ill patients in the intensive care by at least $10 \%$ (13). In a follow-up study of discharged elderly patients who developed delirium during hospitalization, the mortality rate within the 12 months following discharge was found to be $63.3 \%$. In contrast, the mortality rate was $17.4 \%$ for control patients (14).

Complications such as malnutrition, fluid and electrolyte imbalances, aspiration pneumonia, pressure ulcers, weakness, and restless behavior that may result in falling and injuries/ fractures, fidgetiness, and getting lost are frequently observed in medical patients with delirium. In elderly and postoperative patients, it may prolong the hospitalization period, increase complications and cost, and can cause long-term loss of faculties. Delirium in dementia patients reflects the severity of the disorder and results in a decrease in life expectancy.

Pathophysiology: The last common pathway in all forms of organic mental disorders such as delirium and dementia are the alterations in cortical brain functions. These can be as follows: 1) an endogenous or exogenous process that affects the cerebral neurochemical function and 2) physical or structural damage of the cortex. Functional and structural disorders result in the impairment of cognitive functions such as wakefulness, orientation, and memory.

The mechanisms underlying delirium are not yet precisely known. It can develop because of numerous structural or physiological causes. The main hypothesis is the reversible impairment of the cerebral oxidative metabolism and multiple neurotransmitter abnormalities. Subsequent observations supported multiple neurotransmitter abnormalities (15).

Acetylcholine: Animal and clinical studies have revealed that acetylcholine is one of the most important neurotransmitters in the pathogenesis of delirium. It is demonstrated that most of the causes that lead to delirium are accompanied by a decrease in acetylcholine. Anticholinergic drugs are one of the well-known causes of acute confusional states. One of the most common causes of delirium is anticholinergic drugs $(16,17)$. Even numerous drugs that are not considered to be among the classical anticholinergics, such as digoxin, theophylline, and cimetidine, exhibit increased in vitro cholinergic activity. Other numerous commonly used drugs and also over dosage are shown to cause mental state changes. Patients, such as Alzheimer's patients, in whom cholinergic transition is disrupted are especially prone to develop these mental changes. The anticholinergic activity is increased in postoperative delirium patients.

Dopamine: There is a reciprocal relationship in the brain between cholinergic and dopaminergic activity. Symptoms are improved with haloperidol and other dopamine antagonist neuroleptics.

Regarding other relevant neurotransmitters in delirium, human and animal studies have revealed that serotonin levels are increased in hepatic encephalopathy and septic delirium. Hallucinogens such as lysergic acid diethylamide (LSD) act as agonists for serotonin receptors. Furthermore, serotonergic agents can cause delirium. In alcohol withdrawal-induced delirium, the locus coeruleus and hyperactivity of its noradrenergic neurons are involved. Increased GABA levels are also observed in hepatic encephalopathy. Increase in ammonia levels 
in hepatic encephalopathy patients causes increased levels of the glutamate and glutamine amino acids, which are GABA precursors. Decreased levels are observed in delirium patients due to benzodiazepine use and alcohol withdrawal (18).

Cortisols and beta-endorphins: Disruption in cortisol and the circadian rhythm is demonstrated in delirium. This findings support a probable relationship between glucocorticoids and delirium.

Inflammatory mechanisms: Certain recent studies support the roles of cytokines such as interleukin- 1 and interleukin-6, and tumor necrosis factor alpha in delirium (17-20). These factors have been demonstrated to cause cognitive disorders and to cross the blood-brain barrier. An impaired bloodbrain barrier may allow neurotoxic agents and inflammatory cytokines to enter CNS, and this may cause delirium (21). Following numerous infections, inflammation and toxic causes, endogenous interleukin-1 is released from the cells. Head traumas and ischemia are characterized by interleukin-1- and interleukin-6-mediated brain reactions. In some cases, components of infectious agents that are outside the nucleus, such as the GP120 component of the AIDS virus, are shown to cause delirium (22).

Oxidative stress hypothesis: According to this view, any factor that causes a disruption in any of the processes procuring, obtaining, or using that are necessary for oxidative metabolism can cause delirium.

Any condition that degrades the capacity of endogenous reduction systems, which neutralize toxic materials, disrupts the system and a new equilibrium, called "oxidative stress," occurs. Causes such as tissue damage and hypoxia increase oxygen and energy consumption, and reduce the cerebral oxidative metabolic rate, with the resulting cerebral dysfunction causing the symptoms of delirium (15).

Structural mechanisms: The specific neural pathways that cause delirium are unknown. Metabolic encephalopathy imaging studies and visible structural causes (traumatic brain injuries, stroke) support the hypothesis that anatomical pathways play a more important role than others. The main neuroanatomic area that is active in delirium is the reticular formation, which is the primary structure that regulates attention and wakefulness.

The dorsal tegmental pathway, which projects from the mesencephalic reticular formation to the tectum and thalamus, also plays a role in delirium. Normal attention requires the existence and appropriate function of the upper brain stem, the ascending reticular activating system (ARAS) and the cortical polymodal association region. Stimulation of the ARAS increases wakefulness, and its lesions cause sleep, coma, and akinetic mutism.

In the mechanisms of acute confusional states, not only specific structural lesions, but also widespread metabolic causes play a role. Metabolic pathways involving the ARAS and the polymodal cortex are the most sensitive ones in the develop- ment of confusional states because these two regions include the densest polysynaptic chains. Additionally, widespread metabolic causes disrupt acetylcholine transmission and render cortical neurons more sensitive to afferent stimulation. These impairments can also increase the postsynaptic stimulated responses by affecting norepinephrine transmission.

It has also been suggested that brain metabolism generally slows down in delirium. This decreased metabolism is accepted to be compatible with the slowing down occurring in electroencephalography (EEG) and cognitive disorders. There are studies that found a relationship between a decrease in the blood supply to the brain and delirium (23). Despite that, an apparent pathological change could not be observed in the brain of patients that died due to delirium. However, the lesions in these patients are considered to be generally located in the midbrain, subthalamus, and temporal lobe. All these structures are closely related to the ARAS and the limbic system.

Symptoms, criteria, and diagnosis: Some of the patients can display prodromal symptoms such as restlessness, anxiety, irritability, attention deficit or sleep disorders. An increase in anxiety, weakness, headache, torpor, nightmares, and sleeping problems occur at the early stages. A clinical picture of delirium can include symptoms and signs that suggest many other mental illnesses.

DSM-5 (2013), the latest edition of DSM, necessitates the inclusion of the following criteria for the diagnosis of delirium (1):

A. Deficit in attention (decrease in directing, focusing or maintaining alertness, and shifting capability) and awareness (environmental orientation).

B. Disorder develops within a short span of time (hours or days) and fluctuates throughout the day.

C. Other changes in cognitive functions (memory deficits, disorientation, speech disorders).

D. Disorder cannot be better explained with another neurocognitive disorder and it does not occur in a condition where wakefulness is decreased more severely, such as coma.

E. Evidence consisting of history, physical examination, and laboratory findings show that the disorder occurred as a result of a general medical condition, substance intoxication or withdrawal, exposure to a toxin, or the direct physiological result of multiple causes.

DSM-5 also recommends including acute markers for delirium cases that last a couple of hours or days and chronic markers, for delirium cases that last weeks or months.

Types of delirium: 3 subtypes of delirium are defined, based on the degree of alertness and behavioral symptoms. 1. Hyperalert-hyperactive delirium is characterized by overstimulation-excessiveness and mobility. Hallucinations and delusions are seen in patients. Playing with the bed and genital region, 
and trying to leave the bed or the room are commonly observed. For these patients, hospitalization duration is shorter and their prognoses are accepted to be better. Substance intoxication and withdrawal, and drugs such as anticholinergic agents, are reported to be the main cause of hyperactive delirium by affecting the brain neurotransmitter systems. 2. Hypoactive delirium is characterized by drowsiness, clouded consciousness, and inactivity. Processes due to acute diseases such as infections, hypoxia, hypothermia, hyperglycemia, hepatic, and renal failure, and thyroid diseases appear to be the predominant cause of hypoactive delirium. These patients are confused and appear fatigued. 3. In mixed delirium, patients usually exhibit sedation during the day, and agitation and behavioral problems at night. Among intensive care patients, mixed and hypoactive delirium cases are observed the most (24-27).

Diagnostic difficulties: Delirium has a clinical diagnosis, but it does not have a specific test. Obtaining a detailed history, and a careful and complete physical examination, including mental examination, are required. Fever, pulse, blood pressure, and respiratory examinations must definitely be performed. Patients display difficulty in maintaining attention, short-term memory and orientation, and also exhibit insight and judgmental disorders. The key element is the fluctuations in consciousness. Disorientation plays the main role in diagnosis, given that it can be assessed easily. Bedside mental examination and interviews fluctuate dramatically. Therefore, in delirium patients and suspected cases of delirium, these interviews must be performed frequently.

Due to the fact that delirium patients are often confused and cannot provide accurate information, gathering details from family, caregivers and nurses is very important. Nurse notes are especially important for documenting disorientation periods, abnormal behaviors and hallucinations that are observed during night watch. These notes must not be in simple sentences such as 'he was hostile, absent-minded.'

Hypoactive delirium can be mistaken with depression, especially if the patient appears calm. Depression symptoms often occur in delirium. A recent study showed that presence of a dysphoric mood and hopelessness in hospitalized patients increases the risk for developing delirium (28). Especially in elderly patients that suffer dementia or depression, delirium must always be suspected in case of an acute or subacute brain function disorder (29). Delirium is an important cause of psychotic symptoms such as bizarre delusions, and abnormal and aggressive behavior.

Main symptoms: In articles that review studies conducted in the nineties, the most common symptoms are reported as clouding of consciousness (65\%-100\%), difficulty in focusing, and maintaining and shifting attention (62\%-100\%), disorientation (the most common symptom, with $78 \%$ $100 \%)$, disorganized thought (95\%), and short-term memory deficit and recalling problems $(62 \%-90 \%)(30,31)$. The type and prevalence of symptoms vary from patient to patient (32). However, the following symptoms are frequently observed in delirium: a. Changes in consciousness: In hypoactive-type delirium, torpor, and sleepiness are prominent, and awareness is lessened. Patient cannot fully be awoken and can easily enter the sleeping state. In the hyperactive type, however, hyperexcitability and irritability are observed. They may exhibit overalertness and startling reactions with heightened attention.

b. Attention disorders: Difficulty in focusing and maintaining and shifting attention are the most striking symptoms of delirium. Attention assessment can be performed by observing patient's monitoring levels during an interview, examination or inspection. However, attention is examined more objectively by some bedside tests. Digit span test is based on reading out loud 4-6 nonconsecutive numbers and having the patient repeat them back. Continuous performance test is conducted by uttering a letter per second and asking the patients to raise their hands whenever the letter $\mathrm{A}$ is uttered.

c. Disorientation: It is the most common symptom. However, it is not only very important for being common, but also for being the dominant factor in diagnosing delirium. It is particularly indicative in diagnosis, given that individuals can be assessed by asking simple questions regarding where they are and what time it is. In particular, its frequent examination in suspected delirium patients is important in order to discover and treat a potential delirium state early. Disorientation most frequently is related to time (confusing the hour, day, month), but it can also be related to location (patients can say they are at home, when they are at the hospital) and in more severe cases, it can be related to individuals (not being able to identify relatives).

d. Disorganized speech and thought. This consists in an incoherent train of thought where the connections between words, sentences, and topics are weakened. It can appear as cryptic and incoherent speech. Speech disorder can manifest itself as difficulty in naming objects, inability to speak, write, or comprehend speech/writing.

e. Perception and sensory disorders are common. Sensory disorders can occur as illusions (mistaking water pipes in the room for snakes, or the folds on the bed sheets for a mouse) and hallucinations (such as little people going in and out of the room). Illusions and hallucinations are usually visual but they can occur with other senses as well.

f. Both recording and recall memory, as well as recognition memory, are impaired. Near memory is usually affected more than long-term memory.

g. Emotional changes can be periodical, in a way that relatives of the patient interpret them as personality changes. There is no affection or mood feature specific to delirium. However, most of the time disorders occur and these can be manifested as anxiety, anger, apathy, depression, euphoria, and irritability.

h. Unsystematic delusions with a tendency to see the evil (they will kill me, dissect me) that could occur. Misinterpretations (patients can interpret the nurses' attempts to medicate them as trying to poison them) can turn to delusions. 
i. Reversal of the sleep-wake cycle is common. Sleep disorders can be manifested as daytime drowsiness, nighttime restlessness, and inability to sleep.

j. Behavioral changes: This is the most important symptom that makes it difficult to examine delirium patients. Hyperactive delirium patients display increased alertness and hypervigilance. They can be agitated, excessive, and aggressive. Hypoactive patients, by contrast, are sleepy. Inactivity and retardation are prominent.

k. Symptoms fluctuate throughout the day, commonly with a slight improvement during the day and deterioration during the night. Fluctuation and changing of the symptoms throughout the day are among the most typical features of delirium. This feature is undoubtedly one of the most important reasons for overlooking the diagnosis in patients that are not frequently met.

Neurological symptoms: Speech dysphasia (anomia) is mostly observed as systematic misnomers. Frequently there are misnomers that reflect the associations from the individual's past experiences. For example, a patient who is a lumberjack can call a stethoscope pliers. Cerebral dysarthria; tremors, mostly as action tremors; hepatic encephalopathy and uremic asterixis; flapping motion when arms are stretched in front; and various motor abnormalities can be observed.

Physical signs: A complete physical examination must be performed in every patient that exhibits mental state changes. Overall picture, vital signs, hydration state, evidence of physical trauma and neurological signs must be assessed very carefully. A quick bedside neurological examination is very important for a quick diagnosis of delirium, as is the case in all organic mental disorders. If delirium or suspicion of delirium is present, pupillary and funduscopic abnormalities, neck stiffness, goiter, cardiac murmurs or cardiac rhythm disorders must be investigated. Pulmonary auscultation, an abdominal examination that will reveal hepatosplenomegaly or splenomegaly, and a skin examination that will help understand icterus or eruption would be useful. The overall picture can give an idea regarding substance or alcohol abuse. A musty smell and a fruity smell can be indicative of fetor hepaticus and ketoacidosis, respectively. An elevated serum ammonia level along with jaundice or asterixis, can indicate liver failure. Agitation and tremors can suggest sedative or alcohol withdrawal.

Assessment of vital signs in a delirium patient for whom examinations and assessments necessary to establish a diagnosis prove difficult due to excessiveness and restlessness is both easy and it can yield very valuable information. High fever can be related to infections, thyroid storm, aspirin toxicity, or adrenergic acceleration due to high doses or withdrawal of certain substances. Extreme hyperthermia and pinpoint pupils can be observed in pontine strokes. Diabetic ketoacidosis, sepsis, stimulant intoxication, and aspirin overdose must be considered in patients with an increased breathing rate; whereas narcotic overdose and various sedative intoxications must be considered in patients with a decreased breathing rate. Fast pulse rates can be related to fever, sepsis, dehydration, thyroid storm, various cardiac arrhythmias, and drug overdose. Decrease in pulse rate can be indicative of increased intracranial pressure, asphyxia or complete cardiac block. Increased blood pressure due to adrenergic overload occurs very frequently in delirium. Preeclampsia must be definitely evaluated in pregnant women with diastolic blood pressures of $75 \mathrm{~mm} \mathrm{Hg}$ in the second trimester, and of $85 \mathrm{~mm} \mathrm{Hg}$ in the third trimester. Increased intracranial pressure must be investigated in hypertension and bradycardia patients. In a delirium patient with hypotension, differential diagnosis regarding dehydration, diabetic coma, traumatic hemorrhage, aneurysmal rupture or GI bleeding must be conducted. Pupillary dilation can be due to hallucinogens, amphetamines, cocaine, Atropa belladonna intoxication or anticholinergic drugs. Pupillary constriction can be secondary to narcotic intoxication. Serious head traumas are often difficult to identify. Funduscopic examination, and clues such as pupillary inequality can sometimes be helpful.

Laboratory studies: Complete blood count (helps with the diagnosis of infection and anemia), electrolytes (low and high values), glucose (hypoglycemia, diabetic ketoacidosis), kidney and liver function tests (for kidney and liver failure diagnoses), thyroid function tests (hypothyroidism), urinalysis (for urinary infections), substance screening in urine and blood (for toxicological tests), thiamine and vitamin $\mathrm{B}_{12}$ levels (for vitamin deficiency), tests for bacteriological and viral etiologies (for infections), sedimentation, substance screening including alcohol level, HIV tests and, if required, other infection tests must be performed.

Imaging studies: Head computed tomography (CT) and magnetic resonance imaging (MRI) can be helpful for diagnosing structural lesions. In delirium cases due to alcoholsedative withdrawal, rapid increased EEG waves can be observed, whereas in toxic and metabolic delirium cases (hepatic encephalopathy), EEG waves are slow and diffuse. Triphasic waves can be found due to toxicity and metabolic deficiencies, and continuous changes can be found in non-convulsive status epilepticus. Chest radiography is used for diagnosing pneumonia and congestive heart failure.

Measuring and assessment criteria: General medical, psychiatric and neurological history, and examinations are required for diagnosis. Diagnosis is established by assessing the findings that are obtained through interviews. However, numerous criteria have been developed for grading screenings, diagnoses, and symptoms. Periodical assessment of diagnostic criteria and knowing patient's initial state of mind are essential for a proper diagnosis.

Mini-Mental State Examination is a test that can easily provide insight on the severity and nature of the mental state disruption in all organic mental disorders, and not just delirium. A score of less than 24 indicates the presence of another problem affecting delirium, dementia or mental state. In such cases, additional diagnostic procedures that will confirm the possibility of delirium must be performed. 
The clock-drawing test is another test that is sensitive to cognitive disorders and that is easy to perform. Patient is asked to draw a clock, including numbers and hands, and to draw the hands to show, for instance, 8:20. In this test, two or more mistakes are usually indicative of cognitive disorders.

The two most common tests used for screening purposes are the Delirium Symptom Interview (DSI) and the Confusion Assessment Method (CAM) (33, 34). Specificity-sensitivity values of DSI are relatively low. Its effectiveness in deliriumdementia distinction has not been tested. The Confusion Assessment Method, however, is a test which has been proven to be effective for distinguishing between delirium and dementia, has high specificity-sensitivity values, and is also adapted to Turkish (35). It has four parts: 1) acute onset with fluctuations throughout the day; 2) attention deficit (can they remember what has been mentioned? can they focus their attention?); 3) decreased ability for organized thought (meaningless speech, illogical thoughts, digressions) and; 4) changes in consciousness. According to the Confusion Assessment Method, the first two signs must definitely be present in order to establish a delirium diagnosis, and additionally, at least one of the third or fourth signs must be present.

Furthermore, there are various criteria that measure the severity of delirium symptoms. A Turkish adaptation of the Delirium Rating Scale is published. Despite mostly being used for assessing the severity of symptoms, it has been reported that an score of 11-12 can be considered as a cut-off point, and that values above this score can be accepted to be indicative of risk for delirium (36).

Communication with intensive care patients is difficult, therefore observing and recognizing delirium is difficult in high-risk patients. For these patients, criteria that can be applied by non-psychiatrists, such as nurses and anesthesiologists, have been developed.

Causes: When confronted with a case of delirium, the clinician must consider all conditions that may have caused it. Lipowski (1989) defined four main groups: primary cerebral diseases, systemic diseases, intoxication, and psychoactive substance withdrawal (37). The DSM lists the main causes of delirium as the following: 1) substance intoxication delirium; 2) substance withdrawal delirium; 3 ) drug-induced delirium; 4) delirium due to other medical conditions; 5) delirium due to multiple etiologies; 6) other defined types of delirium; 7) unidentified delirium (1).

In sources that examine the conditions that play a role in the development of delirium, it can be found that often the factors that cause the condition are considered together with conditions that enable it. This leads to mixing up and neglecting preventative and therapeutic approaches. Therefore, predisposing, precipitating and causal factors must be considered with a holistic approach.

Predisposing factors: Studies reveal that the major predisposing factors are advanced age, presence of cognitive disor- ity, stroke or depression history, chronic renal or lung failure, dehydration, malnutrition, alcohol or smoke use, and visual/ auditory disorders $(38,39)$.

Dementia is an important risk factor for delirium, and delirium development rates in dementia patients are found to be as high as $22 \%-89 \%$ (40). Conversely, delirium can be a risk factor for dementia and it can also be indicative of dementia. Recent changes in the awareness level and cognitive functions of a dementia patient can be a warning sign. Especially in elderly patients, delirium can be the only symptom of the underlying condition. Patients with infection, myocardial infarction or stroke can show symptoms of delirium without fever, chest pain or hemiparesis, respectively.

Precipitating factors: There are many factors that can precipitate delirium. Drugs, immobilization, urinary catheter use, barrier use that restricts physical activity, dehydration, malnutrition, environmental factors and psychosocial problems are the leading factors. Even though surgery, in and of itself, is not considered to be a risk factor for delirium, many accompanying conditions can precipitate it. Additional preoperative (dementia, polypharmacy, drug withdrawal, fluid-electrolyte imbalance), intraoperative (meperidine, long-acting benzodiazepines, anticholinergics) and postoperative (hypoxia, hypertension) factors can cause delirium due to operations to be commonly observed. Type of anesthesia (general or local) is reported to make no significant difference regarding delirium onset.

Environmental factors such as an unfamiliar environment, disruption in sleeping patterns, frequent room changes, sensory overload or deprivation can especially cause delirium in elderly patients that are already predisposed. Drugs are important risk factors for delirium, and delirium cases due to drugs are especially widespread in hospital settings. This is particularly important in elderly patients. Therefore, drug and substance use history of the patients must be obtained very carefully. In elderly individuals, even therapeutic doses and levels of drugs can sometimes cause delirium.

Psychosocial factors such as depression, pain, psychological stress, loss of independence, or social support are important risk factors for delirium, especially in the age group that these factors are commonly observed, and they are often ignored by doctors.

Most commonly reported medical causes: Almost all medical conditions, intoxications, and drug therapies can cause delirium. A list of these conditions is provided in Table 1.

\section{Medical Care and Treatment Principles:}

Prevention: Due to the additional cost of delirium progression, prevention must be the primary goal. High-risk patients for delirium must be closely monitored during admittance, and medical and surgical operations. Patient's state of mind must be monitored as a vital sign in high-risk patients. Especially, in high-risk patients, orientation, and behavior must be frequently assessed between visits, considering the fluctuations throughout the day. 
Fundamentals of care and treatment: Underlying causes must be investigated when delirium is diagnosed or suspected. Treatment should address the underlying cause. Measures for treating behavioral and psychiatric symptoms include drug therapy, along with environmental control. Currently, the main principles of delirium treatment are summarized as defining the picture and reviewing the possible causes, psychological approach for symptoms, and drug therapy directed at symptoms.

Physical-medical care: Given that the main treatment is directed at the underlying cause, physical examination and laboratory assessments must be performed carefully. Agitation and delirium can sometimes increase by reversible additional causes. Conditions that may cause physical discomfort, such as urinary retention, fecal difficulties, musculoskeletal system pains or extremity ischemias, must be fixed. Non-essential patient care treatments must be terminated (e.g., IV poles). Liquids and food must be handed out with care because patient can be reluctant to eat and a balanced intake may be required. Multivitamins, especially thiamine, must be administered to patients suspected of alcohol toxicity or withdrawal.

Environmental control: The environment for delirium patients must be calm, stable, and well-lit. Excess and lack of stimulants can increase symptoms of delirium. Memory clues such as a calendar, clock, or orientation aids can be useful. Some delirium patients can harm themselves or others, for instance, the patient next to them. Being alone in an unfamiliar environment can exacerbate the symptoms. For these reasons, and due to dangers such as vomit aspiration and IV entanglement, which can be caused by cognitive disorders, and disorientation, delirium patients must never be left alone or without a companion.

Patients and their relatives must be informed about likely causes, importance, progression, and potential consequences of delirium. Families usually worry, thinking that the patient suffers from a severe brain damage or lost his/her mind. Families must be reassured by explaining them that delirium is usually temporary, although it demonstrates the severity of the underlying disease. Relatives and friends might want to visit frequently these patients, and their visits must be encouraged, provided that they fulfill certain conditions and each of them are scheduled for different times.

Due to memory problems, one must reintroduce himself every time he sees the patient. Speaking with a faint and soft voice in a polite manner, as well as explaining the planned procedures and situation clearly can help to reduce anxiety. Instead, of physical limitation, especially constraining, it would be appropriate that a family member stays with the patient to help. In case detection is required, it must be performed carefully. Sensory deficits must be addressed and, if the patient is using glasses and hearing aids, their use must be encouraged.

Drug therapy: Delirium treatment must be primarily directed at the underlying cause. Due to the fact that it is related to the severity of the underlying medical condition, one must act quickly and patient's state must be brought under control. A psychiatrist's help can be sought in case delirium diagnosis is suspected, or for drug selection. However, treatment directed at the main cause will, of course, be conducted in the relevant clinic by the supervising doctor.

Psychotropic drug therapies must be used in cases of severe delirium, where the symptoms prevent the application of the necessary interventions (intubation, IV treatment), and

Table 1. The most commonly observed medical conditions that cause delirium:

\section{Structural changes}

Closed head injuries or brain hemorrhages

Cerebrovascular events such as cerebral infarction, subarachnoid hemorrhage, and hypertensive encephalopathy

Primary or metastatic brain tumors

Brain abscesses

\section{Metabolic causes}

Fluid-electrolyte abnormalities, acid-base imbalances, and hypoxia Hypoglycemia

Hepatic and renal failure

Vitamin deficiency cases (in particular, thiamine, and cyanocobalamin)

Thyroid and parathyroid endocrinopathies

\section{Hypoperfusion states}

Shock

Congestive heart failure

Cardiac arrhythmias

Anemias

\section{Infectious causes}

MSS infections such as meningitis

Encephalitides

Brain infections due to HIV

Septicemia

Pneumonia

Urinary tract infections

\section{Toxic causes}

Substance intoxication-Alcohol, heroin, cannabis, PCP, LSD

Drug-induced delirium

Anticholinergics (Benadryl, TCAs)

Narcotics (Meperidine)

Sedative-hypnotics (Benzodiazepines)

Histamine-2 (H2) blockers (cimetidine)

Corticosteroids

Centrally acting antihypertensives (Methyldopa, reserpine)

Anti-Parkinson's drugs (Levodopa)

Substance withdrawal due to alcohol, opioids, and benzodiazepines 
where the safety of the patient and others is at risk. Clinicians must bear in mind that there is no ideal drug in the treatment of delirium symptoms, but any alternative can further deteriorate the mental state and it can prevent tracking mental state changes. If a drug therapy is started, it should be started with the minimum dose and for the shortest possible duration. Dose must be slowly reduced as the symptoms are brought under control.

Pharmacological treatment of delirium helps the safety of the patient and clinic personnel by reducing anxiety and agitation. Sedation is not necessary in hypoactive delirium cases; environmental control and interventions directed at the underlying cause are essential, but low doses of antipsychotics can be useful in cases of disturbing hallucinations. Psychotropic drugs are used in hyperactive delirium for behavioral symptoms such as excessiveness, aggressiveness, restlessness, sleeplessness, and for psychotic symptoms such as hallucinations and delusions.

Antipsychotics: This is the drug of choice in the treatment of patients with advanced hyperactive or psychotic symptoms. Old class, high-potency antipsychotics such as haloperidol are suitable drugs, but they have neurological side effects. New antipsychotics such as risperidone, olanzapine, quetiapine reduce symptoms with less side effects. Low doses must be used in elderly patients, and doses must be slowly reduced as the symptoms are brought under control. Evidence regarding the use of antipsychotics for treatment of intensive care patients is currently insufficient (40).

Among the recommended antipsychotic drugs, haloperidol remains to be the first choice in treatment guidelines, showing effectiveness in treatment, a good receptor profile, safety, and applicability in different forms (41). In a Cochrane review conducted by Lonergan et al. (40), haloperidol was demonstrated to reduce the degree and duration of delirium in postoperative cases, and to have a direct effect on delirium progression, rather than just treating behavioral symptoms. Haloperidol causes orthostatic hypotension less frequently than other typical antipsychotics, and it has fewer anticholinergic side effects. However, extrapyramidal side effects and dystonia are more commonly observed. In particular, higher doses can lead to falling. Even though it is recommended that haloperidol is started with lower doses, such as 1-2 mg, and that it should be administered every $2-4$ hours if necessary, there are studies that show that a high-dose bolus and intravenous (IV) administration can be more effective and reduce complications due to repeated dosage (42). However, it must be remembered that the FDA has not approved intravenous administration yet for haloperidol, and that IV administration is twice as potent as oral administration. Also, the clinician should also bear in mind that antipsychotics increase the risk for cardiac dysrhythmia and QT prolongation.

The use of atypical antipsychotics in the treatment of delirium has been discussed in recent years. There are case reports claiming that delirium can be effectively treated with atypical agents such as risperidone, olanzapine, quetiapine (43-45). In an article that reviews studies performed between 1996 and 2008, Pelland and Trudel (45) report that atypical agents are as effective as haloperidol, and also safer, but that oral, IM and IV forms of haloperidol remain to be a tried-and-tested gold standard, especially for intensive care patients.

The risk for stroke must be assessed appropriately, especially in patients with cardiovascular and cerebrovascular risk factors, given that there is some evidence suggesting that all antipsychotics can cause them. There are reports of antipsychotics causing ischemic stroke and atypical antipsychotics causing QT prolongation, and increasing the risk for developing sudden cardiac death and pneumonia $(46,47)$. Antipsychotic usage is reported to increase mortality risk in American nursing homes, which also house elderly delirium patients. This risk increase is found to be the most for haloperidol and the least for quetiapine (48). These findings reveal that antipsychotics must be administered in low doses and for short durations.

In conclusion, antipsychotics must be only used in severely agitated, restless or potentially harmful (to themselves or others) patients, and they must be used for the shortest possible duration and at the lowest effective dose. In all cases, antipsychotics must only be used if behavioral interventions are not possible or successful, and the progression must be closely monitored (49).

Benzodiazepines: These are used in seizures or sedativehypnotic withdrawal cases. In other cases, it is only used in addition to neuroleptic treatment. Studies that compare haloperidol+benzodiazepine treatment (combined therapy) with haloperidol treatment (monotherapy) report fewer extrapyramidal symptoms (EPS) in patients receiving the combined treatment (50). In such cases, it reduces the dose of neuroleptic that is used to control agitation and, therefore, reduces EPS. Benzodiazepines can also be preferred for treating unknown substance usage. One must proceed with caution, as it can cause respiratory depression, especially in patients with respiratory problems or weak patients. Lorazepam is the drug of choice, due to being short-acting and not having active metabolites.

Vitamins: Vitamins such as thiamine and cyanocobalamin are used to treat vitamin deficiencies, alcohol withdrawal and Wernicke's encephalopathy. It must definitely be administered in suspected cases of delirium.

\section{Conclusion}

Delirium is a syndrome that causes disruption in the brain function, develops due to numerous medical conditions, and manifests itself through various cognitive disorders, primarily related to consciousness. There is a high incidence of delirium in internal and surgical clinic patients, and it is a red flag that indicates that the underlying medical condition is very severe. Frequent examinations of orientation and attention levels in predisposed patients can make diagnosis easier. Treatment must be primarily directed at the underlying medical condition and must be provided by the supervising clinic. However, the importance of non-pharmacological approaches must not be forgotten. 
Peer-review: Externally peer-reviewed.

Conflict of Interest: No conflict of interest was declared by the authors.

Financial Disclosure: The authors declared that this study has received no financial support.

\section{References}

1. American Psychiatric Association. Diagnostic and Statistical Manual of Mental Disorders. Fifth Edition. Arlington, VA, American Psychiatric Association, 2013.

2. Ely EW, Margolin R, Francis J, May L, Truman B, Dittus R, et al. Evaluation of delirium in critically ill patients: validation of the Confusion Assessment Method for the Intensive Care Unit (CAM-ICU). Crit Care Med 2001; 29: 1370-9. [CrossRef]

3. Caine ED, Lyness JM. Delirium, dementia and amnestic and other cognitive disorders. In Sadock B, Sadock V, eds. Comprehensive Textbook of Psychiatry. Philadelphia, Lippincott Williams \& Wilkins, 2000. p. 854-924.

4. Inouye S, Bogardus S, Leo-Summers L, Acampora D, Holford T, Cooney L. A multicomponent intervention to prevent delirium in hospitalized older patients. N Engl J Med 1999; 340: 669-76. [CrossRef]

5. Weinberger MW, Carnes M. Diagnosis and management of delirium. Prim Care Update Ob/Gyns 1997; 4: 84-126. [CrossRef]

6. Berrios GE. Delirium and confusion in the $19^{\text {th }}$ century. Br J Psychiatry 1981; 139: 439-49. [CrossRef]

7. Schneider F, Böhner H, Habel U, Salloum JB, Stierstorfer A, Hummel TC, et al. Risk factors for post operative delirium in vascular surgery. Gen Hosp Psychiatry 2002; 24: 28-34. [CrossRef]

8. Litaker D, Locala J, Franco K, Bronson DL, Tannous Z. Preoperative risk factors for postoperative delirium. Gen Hosp Psychiatry, 2001; 23: 84-9. [CrossRef]

9. van der Mast RC, van den Broek WW, Fekkes D, Pegglinkhuizen L, Habbema JD. Incidence of and preoperative predictors for delirium after cardiac surgery. J Psychosomatic Res 1999; 46: 479-83. [CrossRef]

10. Gagnon P, Allard P, Masse B, DeSerres M. Delirium in terminal cancer: a prospective study using daily screening, early diagnosis and continuous monitoring. J Pain Symptom Manage 2000; 19: 412-26. [CrossRef]

11. Sandberg O, Gustafson Y, Bransström B, Bucht G. Prevalence of dementia, delirium and psychiatric symptoms in various care settings for the elderly. Scand J Soc Med 1998; 26: 56-62. [CrossRef]

12. O'Keeffe S, Lavan J. The prognostic significance of delirium in older hospital patients. J Am Geriatr Soc 1997; 45: 174-8. [CrossRef]

13. Klouwenberg PM, Zaal IJ, Spitoni C, Ong DSY, van der Kooi AW, Bonten BJM, et al. The attributable mortality of delirium in critically ill patients: prospective cohort study. BMJ 2014; 349: g6652. [CrossRef]

14. McCusker J, Cole M, Abrahamowicz M, Primeau F, Belzile E. Delirium predicts 12-month mortality. Arch Intern Med 2002; 162: 457-63. [CrossRef]

15. Maldonado JR. Neuropathogenesis of Delirium: Review of Current Etiologic Theories and Common Pathways. Am J Geriatr Psychiatry 2013; 21: 1190-222. [CrossRef]

16. Flacker JM, Wei JY. Endogenous anticholinergic substances may exist during acute illness in elderly medical patients. J Gerontol Med Sci 2001; 56: 353-55. [CrossRef]

17. Han L, McCusker J, Cole M, Abrahamoqicz M, Primeau F, Elie M. Use of medications with anticholinergic effect predicts clinical severity of delirium symptoms in older medical inpatients. Arch Intern Med 2001; 161: 1099-105. [CrossRef]

18. van Munster BC, Bisschop PH, Zwinderman AH, Korevaar JC, Endert E, Wiersinga WJ, et al. Cortisol, interleukins and S100B in delirium in the elderly. Brain Cogn 2010; 74: 18-23. [CrossRef]

19. Cavallazzi R, Saad M, Marik PE. Delirium in the ICU: an overview. Annals of Intensive Care 2012; 27; 2: 49.

20. Holmes C, El-Okl M, Williams AL, Cunningham C, Wilcockson D, Perry VH. Systemic infection, interleukin 1 beta, and cognitive decline in Alzheimer's disease. J Neurol Neurosurg Psychiatr 2003;74: 788-99. [CrossRef]

21. Banks WA, Farr SA, La Scola ME, Morley JE. Intravenous human interleukin-1 alpha impairs memory processing in mice: dependence on blood-brain barrier transport into posterior division of the septum. J Pharmacol Exp Ther 2001; 299: 536-41.

22. Farr SA, Banks WA, Uezu K, Freed EO, Kumar VB, Morley JE. Mechanisms of HIV type 1-induced cognitive impairment: evidence for hippocampal cholinergic involvement with overstimulation of the VIPergic system by the viral coat protein core. AIDS Res Hum Retrovi 2002; 18 : 1189-95. [CrossRef]

23. Yokota H, Ogawa S, Kurokawa A, Yamamoto Y. Regional cerebral blood flow in delirium patients. Psychiatr Clin Neurosci 2003; 57: 337-9. [CrossRef]

24. Rummans TA, Evans JM, Krahn LE, Fleming KC. Delirium in elderly patients: evaluation and management. Mayo Clinic Practice 1995; 70 : 989-98. [CrossRef]

25. Rudberg MA, Pompei P, Foreman MD, Ross RE, Cassel CK. The natural history of delirium in older hospitalised patients: a syndrome of heterogeneity. Age Ageing1997; 26: 169-74. [CrossRef]

26. Justic M. Does "ICU psychosis" really exist? Critical Care Nurse 2000; 20: 28-37.

27. Peterson JF, Pun BT, Dittus RS, Thomason JW, Jackson JC, Shintani AK, et al. Delirium and its motoric subtypes: a study of 614 critically ill patients. J Am Geriatr Soc 2006; 54: 479-84. [CrossRef]

28. McAvay GJ, Van Ness PH, Bogardus ST Jr, Zhang Y, Leslie DL, LeoSummers LS. Depressive symptoms and the risk of incident delirium in older hospitalized adults. J Am Geriatr Soc 2007; 55: 684-91. [CrossRef]

29. Flaherty JH, Morley JE. Delirium: a call to improve current standards of care. J Gerontol A Biol Sci Med Sci 2004; 59: 350-4 . [CrossRef]

30. Meagher DJ, Trzepacz PT. Delirium phenomenology illuminates pathophysiology, management, and course. J Geriatr Psychiatry Neurol 1998; 11: 150-6. [CrossRef]

31. Trzepacz PT. Update on neuropathogenesis of delirium. Dement Geriatr Cogn Disord 1999; 10: 330-4. [CrossRef]

32. Bhat R, Rockwood K. Delirium as a disorder of consciousness. J Neurol Neurosurg Psychiatry 2007; 78: 1167-70. [CrossRef]

33. Inouye SK, van Dyck CH, Alessi CA. Clarifying confusion: the confusion assessment method. A new method for detection of delirium. Ann Intern Med 1990; 113: 941-8. [CrossRef]

34. Trzepacz PT, Baker RW, Greenhouse J. A symptom rating scale for delirium. Psychiatry Research 1988; 23: 89-97. [CrossRef]

35. Akıncı SB, Rezaki M, Özdemir H, Çelikcan A, Kanbak M, Yorgancı K, et al. Yoğun Bakım Ünitesinde Konfüzyon Değerlendirme Ölçeğinin Geçerlik Güvenilirlik Çalışması. Türk Anest Rean Der Dergisi 2005; 33: 333-41

36. Aydemir Ö, Noyan A, Gülseren AŞ, Kayahan B, Bodur Z, Elbi Hi et al. Deliryum Derecelendirme Ölçeği' nin geliştirilmesi, güvenilirliği ve geçerliliği. Psikiyatri Psikoloji Psikofarmakoloji Dergisi 1998, 6: 21-7.

37. Lipowski ZJ. Delirium in the elderly patient. New England Journal of Medicine 1989; 320: 578-82. [CrossRef]

38. Pisani MA, Murphy TE, Van Ness PH, Araujo KL, Inouye SK. Characteristics associated with delirium in older patients in a medical intensive care unit. Arch Intern Med. 2007; 167: 1629-34. [CrossRef]

39. Fick DM, Agostini JV, Inouye SK. Delirium superimposed on dementia: a systematic review. J Am Geriatr Soc 2002; 50: 1723-32. [CrossRef]

40. Lonergan E, Britton AM, Luxenberg J, Wyller T. Antipsychotics for delirium. Cochrane Database Syst Rev 2007; CD005594 [CrossRef]

41. American Psychiatric Association. Practice guidelines for the treatment of patients with delirium. Washington DC, American Psychiatric Association 1999.

42. Levenson JL. High-dose intravenous haloperidol for agitated delirium following lung transplantation Psychosomatics 1995; 36: 66-8. [CrossRef]

43. Breitbart W, Tremblay A, Gibson C. An open trial of olanzapine for the treatment of delirium in hospitalized cancer patients. Psychosomatics 2002; 43: 175-82. [CrossRef] 
44. Devlin JW, Roberts RJ, Fong JJ, Skrobik Y, Riker RR, Hill NS, et al. Efficacy and safety of quetiapine in critically ill patients with delirium: A prospective, multicenter, randomized, double-blind, placebo-controlled pilot study. Crit Care Med 2010; 38: 419-27. [CrossRef]

45. Pelland C, Trudel JF. Atypical antipsychotic efficacy and safety in managing delirium: A systematic review and critical analysis Psychol Neuropsychiatr Vieil 2009; 7: 109-19.

46. Setoguchi S, Wang PS, Brookhart AM, Canning CF, Kaci L, Schneeweiss S: Potential causes of higher mortality in elderly users of conventional and atypical antipsychotic medications. J Am Geriatr Soc 2008; 56: 1644-50. [CrossRef]

47. Knol W, van Marum RJ, Jansen PA, Souverein PC, Schobben AF, Egberts AC: Antipsychotic drug use and risk of pneumonia in elderly people. J Am Geriatr Soc 2008; 56: 661-666. [CrossRef]
48. Huybrechts KF, Gerhard T, Crystal S, Olfson M, Avorn J, Levin R, et al. Differential risk of death in older residents in nursing homes prescribed specific antipsychotic drugs: population based cohort study. BMJ 2012; 344: e977. [CrossRef]

49. Menza MA, Murray GB, Holmes VF, Rafuls WA. Controlled study of extrapyramidal reactions in the management of delirious, medically ill patients: intravenous haloperidol versus intravenous haloperidol plus benzodiazepines. Heart Lung 1988; 17: 238-41.

50. The American Geriatrics Society Expert Panel on Postoperative Delirium in Older Adults. American Geriatrics Society Abstracted Clinical Practice Guideline for Postoperative Delirium in Older Adults, J Am Geriatr Soc. 2015; 63: c142-150. [CrossRef] 